\title{
Surgery for ischemic colitis: outcome and risk factors for in-hospital mortality
}

\author{
Jörg Genstorfer • Juliane Schäfer • Christoph Kettelhack • \\ Daniel Oertli • Rachel Rosenthal
}

Accepted: 14 December 2013 / Published online: 15 January 2014

(C) Springer-Verlag Berlin Heidelberg 2014

\begin{abstract}
Purpose Surgery for ischemic colitis is associated with high perioperative morbidity and mortality, but the risk factors for mortality and major surgical complications are unclear. Methods In this retrospective single institution cohort study of all patients undergoing colorectal surgery for histologically proven ischemic colitis between 2004 and 2010, we evaluated surgical outcomes and risk factors for in-hospital mortality and major surgical complications.
\end{abstract}

Parts of the data have been presented at the annual meeting of the Swiss Society of Visceral Surgery and of the Swiss Society of Gastroenterology held in Interlaken, Switzerland, 20-21 September 2012.

1. What is current knowledge

- There is little and contradictory knowledge about perioperative risk factors for mortality and surgical complications in patients undergoing surgery for ischemic colitis.

- If surgery is necessary for acute ischemic colitis, it is associated with high perioperative morbidity and mortality of up to $50 \%$.

2. What is new here

- In-hospital mortality is significantly elevated in patients with right-sided or pan-colonic ischemia and after cardiac or aortic surgery.

- There is evidence that patients with ischemic alterations at the resection margin of the histological specimen are more likely to experience major postoperative surgical complications.

Electronic supplementary material The online version of this article (doi:10.1007/s00384-013-1819-1) contains supplementary material, which is available to authorized users.

J. Genstorfer · J. Schäfer $\cdot$ C. Kettelhack $\cdot$ D. Oertli $\cdot$ R. Rosenthal Department of Surgery, University Hospital Basel, Basel,

Switzerland

J. Schäfer

Basel Institute for Clinical Epidemiology and Biostatistics, University Hospital Basel, Basel, Switzerland

J. Genstorfer $(\bowtie)$

Department of Visceral Surgery, University Hospital Basel, Spitalstrasse 21, 4031 Basel, Switzerland

e-mail: jgenstorfer@gmx.de
Results For the 100 patients included in the study, in-hospital mortality was $54 \%$; major surgical complications, defined as anastomotic leakage or rectal stump and stoma complications, occurred in $16 \%$. In the multivariable analysis, hospital death was more likely in patients with right-sided (odds ratio [OR] 3.8; $95 \%$ confidence interval [CI] $1.2,12 ; P=0.022)$ or pancolonic ischemia (OR 11; $95 \%$ CI 2.8, 39; $P<0.001)$, both relative to left-sided ischemia. Decreased preoperative $\mathrm{pH}$ level (OR 2.5 per 0.1 decrease; $95 \%$ CI 1.5, 4.1; $P<0.001$ ) and prior cardiac or aortic surgery (OR 2.4; $95 \%$ CI 0.82, 6.8; $P=0.109)$ were further important risk factors for in-hospital mortality. Major postoperative surgical complications were more likely in patients with ischemic alterations at the resection margin of the histological specimen (OR 3.7; $95 \%$ CI 1.2, $11 ; P=0.022$ ).

Conclusions Colonic resection for ischemic colitis is associated with high in-hospital mortality, especially in patients with right-sided or pan-colonic ischemia. In patients developing acidosis, early laparotomy is essential. Since resection margins' affection seems to be underestimated upon surgery, resections should be performed wide enough within healthy tissue.

Keywords Surgery $\cdot$ Ischemic colitis $\cdot$ Outcome $\cdot$ Risk factors $\cdot$ Mortality

\section{Introduction}

Colon ischemia is the most common type of gastrointestinal ischemia $[1,2]$. The incidence of colon ischemia in the general population remains unknown, but it is supposed to account for 1 in 1,000 hospitalizations in the USA [3, 4]. The clinical findings in patients suffering from ischemic colitis (IC) are highly variable, making diagnosis and clinical management very demanding [5]. The primary challenge in the treatment of 
patients with IC therefore resides in the timely diagnosis. Studies to date focusing on the inpatient population make clear that it is very likely that many cases of colon ischemia are misdiagnosed as inflammatory bowel disease [6].

The majority of colon ischemia is segmental and rarely leads to necrosis and perforation. A chronic fibrosis of the colon wall may eventually trigger stenosis especially in the descending colon near the splenic flexure [7] and then make surgery necessary after months or years. Nevertheless, IC is a relatively common surgical entity, yet large series of patients and multicenter studies are missing. If surgery is necessary for acute IC, it is associated with high perioperative morbidity and mortality of up to $50 \%$ [8-10]. Therefore, the second major challenge is the improvement of surgical outcome in these patients.

There is little and contradictory knowledge about perioperative risk factors for mortality and surgical complications in patients with IC. In a retrospective study of 187 patients undergoing surgery for acute mesenteric infarction, the multivariable analysis revealed time until surgery, markedly elevated leucocytes, and serum lactate level as prognostic factors [11]. Retrospective reports have indicated that occlusion or hypotension in vessel arcades of the right compared to the left colon leads to a worse outcome with higher morbidity and mortality rates [12-14]. Based on a prospective database involving 177 patients, a risk score between 0 and 5 was developed counting one point per present factor out of five factors (acute renal failure, low output heart failure, subtotal colectomy, serum lactate $>2.5 \mathrm{mmol} / \mathrm{l}$, pre- and intraoperative administration of catecholamines). An increasing score was significantly associated with higher mortality. Additionally, predictive variables for impaired long-term survival were investigated with multivariable analysis, revealing mesenteric atherosclerosis to be the only independent risk factor, whereas the other investigated factors age, gender, comorbidity, postoperative morbidity, occlusion of mesenteric artery, low output heart failure, hemicolectomy, and subtotal colectomy were not independent risk factors [14]. Two years before, the same authors published a subset of the series involving 85 patients. In this study, the multivariable analysis indicated risk factors for mortality after surgery for IC different from the ones listed above (ASA [American Society of Anesthesiologists] score $>3$, emergency operation, and blood loss) [15]. No study so far could identify a single risk factor or a combination of risk factors prompting earlier surgery in patients with IC. It is however widely accepted that prolonged time lapse between first symptoms (probable onset of ischemia), clinical detection, and laparotomy may be the key risk factor for mortality in this devastating disease. However, in critically ill patients developing colonic ischemia, e.g., intensive care unit patients after aortic or cardiac surgery, early diagnosis may be very challenging. The goal of the present study was to evaluate the outcomes after surgery for IC and to identify independent important risk factors for in-hospital mortality and surgical complications after surgery for IC. To the best of our knowledge, this is the first study taking into account ischemic alterations at the resection margin of the histological specimen as potential risk factor for surgical complications.

\section{Methods}

Patients

In this retrospective cohort study, we evaluated all patients with colorectal resections for histologically proven IC performed in a 7-year interval between 1 January 2004 and 31 December 2010 at the Department of Visceral Surgery, University Hospital Basel, Basel, Switzerland. Exclusion criteria were ischemia caused by incarcerated hernias, volvulus, abdominal adhesions, concurrent colorectal or pelvic cancer (e.g., gynecological cancers), inflammatory bowel diseases, such as Crohn's disease and ulcerative colitis, and other causes of bowel inflammation but ischemia. Patients for whom there were ambiguous histological findings with doubts whether ischemia was present were excluded.

This study was approved by the local ethics committee, as required for retrospective studies according to local guidelines, in March 2012.

\section{Data collection}

Data were retrieved from the digital patient records in the clinical information system of the University Hospital Basel.

The primary outcome of this study was in-hospital mortality. Secondary outcomes were (a) major postoperative surgical complications, defined as anastomotic leakage and stoma complications or rectal stump insufficiency in patients undergoing resection with primary anastomosis and those undergoing Hartmann's resection, respectively, and (b) other surgical complications such as surgical site infections (SSI) and bleeding/hematoma requiring surgical revision. In addition, we collected nonsurgical complications, such as sepsis/ systemic inflammatory response syndrome (SIRS), peritonitis, pneumonia, urine catheter-associated bloodstream infection, acute renal failure with need for dialysis, and perioperative cerebrovascular insult (CVI).

We collected patients' baseline characteristics such as age, gender, hypertension, morbide adipositas (defined as body mass index $>30$ ), coronary artery disease (CAD), history of myocardial infarction, chronic obstructive pulmonary disease (COPD), atrial fibrillation, chronic renal disease, chemotherapy, prior thromboembolic events of mesenteric arteries (diagnosed in CT scan), prior cardiac or aortic surgery, and the preoperative ASA classification. 
Preoperative laboratory parameters such as lactate, phosphate, leukocyte, C-reactive protein (CRP), and pH level were extracted from patients' charts.

Characteristics of the interventions were collected, such as type of operation, site of resection (left-sided, right-sided, and total colon resection), urgency status, and intraoperative catecholamine administration. Twelve different board-certified surgeons were involved in the operations. Operations were performed as Hartmann resections or resections with primary anastomosis in combination with a left or right hemicolectomy or a total colectomy. Loop ileostomies were not performed. In eight cases, resection was performed with initial blind closure and reanastomosis in a second look operation. It was documented if parts of the small bowel had to be additionally resected. One patient was operated with primary anastomosis and subsequently reoperated because of anastomotic leakage and treated with a Hartmann procedure. Unfortunately, the patient thereafter developed a stoma necrosis of the descending colon. This patient was considered in our analyses in the primary anastomosis group only, since this corresponds to the initial procedure. Finally, we evaluated all histological reports with regard to ischemic alterations at the resection margin.

\section{Statistical analyses}

To evaluate risk factors for in-hospital mortality and major postoperative surgical complications, we used multivariable logistic regression including the most important candidate predictors as prespecified by our study team prior to data analysis. For in-hospital mortality, these were age, gender, preoperative $\mathrm{pH}$ level, side of ischemia, and prior cardiac or aortic surgery, and for major postoperative surgical complications, we considered age and the presence of ischemic alterations at the resection margin of the histological specimen as candidate predictors. Note that the number of hospital deaths respectively major postoperative surgical complications in this study was the limiting factor for the number of candidate predictors to be considered [16].

We used multivariable fractional polynomials (MFPs), combining variable selection with backward elimination (BE) with a rigorous function selection procedure (FSP), to build a multivariable model in the presence of continuous predictors $[17,18]$. Our goal was to include stronger predictors in the model and to identify plausible functions for the continuous predictors (age and preoperative $\mathrm{pH}$ level). For these purposes, the two tuning parameters of the MFP approach, which are nominal $P$ values for selecting a variable with BE and for determining a functional form using the FSP, were set to some less conservative value, namely 0.2 .

To assess the stability of the MFP model with variable selection and transformation, we carried out a bootstrap analysis [19]. The aim of this analysis was to explore whether the final model may depend on a small fraction of the data and to find important stable predictors, with a reasonable functional form for continuous covariates.

Since patients undergoing surgery for IC may either die in the hospital or be discharged alive, we plotted the cumulative incidence functions of these two competing events in patient groups defined by the candidate predictors of in-hospital mortality. For the two continuous variables age and $\mathrm{pH}$ level, we a priori defined cut points. For age, faced with an overall elderly population, we used the median as cut point (i.e., $<74$ versus $\geq 74$ years), and for the $\mathrm{pH}$ level, we assessed patients with $\mathrm{pH} \geq 7.38$ versus those with $\mathrm{pH}<7.38$ (the cut point used to define the presence or absence of acidosis by our central laboratory).

In an additional analysis, we further studied the competing risks hospital death and discharge alive using proportional cause-specific hazards models [20]. There, it is assumed that each cause-specific hazard follows a separate Cox model [21], and for each risk factor, both cause-specific hazard ratios are interpreted simultaneously. Risk factors considered in this analysis were age, gender, preoperative $\mathrm{pH}$ level, side of ischemia, and prior cardiac or aortic surgery.

We used Stata version 10.1 (StataCorp, College Station, TX: StataCorp LP) and the Stata program mfpboot (author: Patrick Royston, available from http://www.homepages.ucl. ac.uk/ ucakjpr/; accessed 20 November 2012) for our main analyses, and for graphics and the additional analysis, we used $\mathrm{R}$ version 2.15.2 (R Foundation for Statistical Computing, Vienna, Austria) and the $\mathrm{R}$ add-on packages cmprsk version 2.2-2 and survival version 2.36-14.

\section{Results}

\section{Patients' characteristics}

Between 1 January 2004 and 31 December 2010, 100 patients underwent surgery for IC. Forty (40\%) patients were female, and the median age of all patients was 74 years (interquartile range [IQR] 67, 79). Many patients had multiple systemic diseases, reflected by an ASA score of $>3$ in $16(16 \%)$ patients; most common were hypertension (72\%), chronic renal disease (54\%), CAD (53\%), and COPD (29\%).

Seventeen $(17 \%)$ patients had prior cardiac surgery; 16 (16\%) patients were operated on the aorta prior to the ischemic event of the colon. The median time between aortic or cardiac surgery and colon resection was 5 days (IQR 2, 13; range 1,60$)$. A prior thromboembolic event in either the superior or inferior mesenteric artery documented by CT scan occurred in $13(13 \%)$ patients. Atrial fibrillation was a known comorbidity in $30(30 \%)$ patients. Acute myocardial infarction 1-2 days prior to colon ischemia was noted in three $(3 \%)$ patients. 


\section{Procedure characteristics}

Eighty ( $80 \%$ ) of the operations were emergency operations conducted within $6 \mathrm{~h}$ of decision for surgery. Four (4\%) patients had laparoscopic surgery. Forty (40\%) operations were left-sided, 33 (33\%) right-sided, and 27 (27\%) were total colon resections because of left, right, and pan-colonic ischemia, respectively. Sixty-two (62\%) operations were discontinuity resections; 38 (38\%) were carried out with primary anastomosis.

All patients received antibiotic prophylaxis 30-70 min prior to surgery using second-generation cephalosporin and metronidazole in combination. When antibiotic therapy was necessary (gut perforation, peritonitis, or surgery- and hospitalrelated complications), duration of antibiotic application was extended and different antibiotics with a broader spectrum were used. Handheld Doppler or other devices to measure macro- and microperfusion were not used.

\section{Outcome after surgery for IC}

Fifty-four (54\%) patients who underwent surgery for IC died in the hospital. For them, the median time of surgery until death was 7 days (IQR 1, 20; range 0,190 ). Of the 62 patients with discontinuity resections, 36 (58\%) died, compared to 18 (47\%) of 38 patients with a primary anastomosis. Forty-six (46\%) patients were discharged alive from the hospital, with a median time of surgery until discharge of 20 days (IQR 13, 49; range 6,126$)$. Descriptive results of surgical and nonsurgical complications, reoperation, and readmission for all 100 patients are shown in Table 1.

Major postoperative surgical complications occurred in 16 $(16 \%)$ patients, out of which anastomotic leakage in 8 patients $(8 / 38 ; 21 \%)$ and rectal stump or stoma complications in 8 patients $(8 / 62 ; 13 \%)$. SSI occurred in $24 \%$, bleeding requiring surgical revision in $14 \%$. Thirty-six patients needed surgical revision because of a complication. In 13/16 (81\%) patients with major postoperative surgical complications, intraoperative vital resection margins with pulsatile bleeding from marginal arteries were documented. In the remaining three reports, the macroscopic appearance was not explicitly mentioned. In two of these three patients, a planned second look with follow-up resection was documented. In the subset of patients with ischemic alterations at the resection margin $(n=26)$, the percentage of second look surgery was higher $(6 / 26,23 \%)$ than in the patients without ischemic alterations at the resection margin $(n=74)(4 / 74,5 \%)$. Out of the six patients with ischemic alterations undergoing a second look, four received a follow-up resection and two did not. Out of four patients without ischemic alterations at the resection margin undergoing a second look, only one patient received a follow-up resection of parts of the small gut. Figure 1 provides a flow chart of patients and their respective
Table 1 Outcomes of patients after surgery for ischemic colitis (IC)

\begin{tabular}{ll}
\hline Outcome & All patients $(n=100)$ \\
\hline In-hospital mortality, \% & 54 \\
Surgical site infection ${ }^{\mathrm{a}}, \%$ & 24 \\
Bleeding/hematoma requiring surgical revision, \% & 14 \\
Anastomotic leakage ${ }^{\mathrm{b}}, \%$ & 21 \\
Rectal stump or stoma complications ${ }^{\mathrm{c}}, \%$ & 13 \\
Pneumonia, \% & 16 \\
Urine catheter-associated bloodstream infection, \% & 2 \\
Sepsis/SIRS, \% & 35 \\
Intraoperative catecholamine administration, \% & 51 \\
Cerebrovascular insult, \% & 14 \\
Reoperation, \% & \\
$\quad$ Elective second look & 10 \\
$\quad$ Complication & 36 \\
Readmission for complication, \% & 8 \\
\hline
\end{tabular}

SIRS systemic inflammatory response syndrome

${ }^{\text {a }}$ Defined according to CDC

${ }^{\mathrm{b}}$ Applicable in patients with primary anastomosis $(n=38)$

${ }^{\mathrm{c}}$ Applicable in patients with discontinuity resection $(n=62)$

outcomes. Frequent hospital-acquired complications were pneumonia (16\%) and CVI (14\%).

Risk factors for in-hospital mortality

Characteristics of patients undergoing surgery for IC grouped by hospital death are shown in Table 2. Relative to patients who were discharged alive from the hospital, patients who died in the hospital had a decreased preoperative $\mathrm{pH}$ level and were more likely to suffer from right-sided or pan-colonic ischemia. Additional patients' characteristics are shown in Supplementary Table 1a, where patients who died in the hospital showed an increased preoperative lactate and CRP level and an increased ASA score. Patients who died in the hospital were more likely to have sepsis/SIRS, peritonitis, an emergency operation, small gut ischemia, and acute renal failure and to require intraoperative catecholamine administration.

In the multivariable analysis, hospital death was more likely in patients with right-sided (odds ratio [OR] 3.8; $95 \%$ confidence interval $[\mathrm{CI}] 1.2,12 ; P=0.022)$ or pan-colonic ischemia (OR 11; $95 \%$ CI 2.8, 39; $P<0.001$ ), both relative to left-sided ischemia, with decreased preoperative $\mathrm{pH}$ level (OR 2.5 per 0.1 decrease; $95 \%$ CI 1.5, 4.1; $P<0.001$ ) and with prior cardiac or aortic surgery (OR 2.4; $95 \% \mathrm{CI} 0.82,6.8 ; P=$ 0.109) (Table 3). Age and gender were not found to be independent important predictors for hospital death in patients undergoing surgery for IC.

The bootstrap analysis confirmed that the preoperative $\mathrm{pH}$ level is an important continuous predictor of hospital death, since it was selected in 195 (98\%) of the 200 bootstrap 


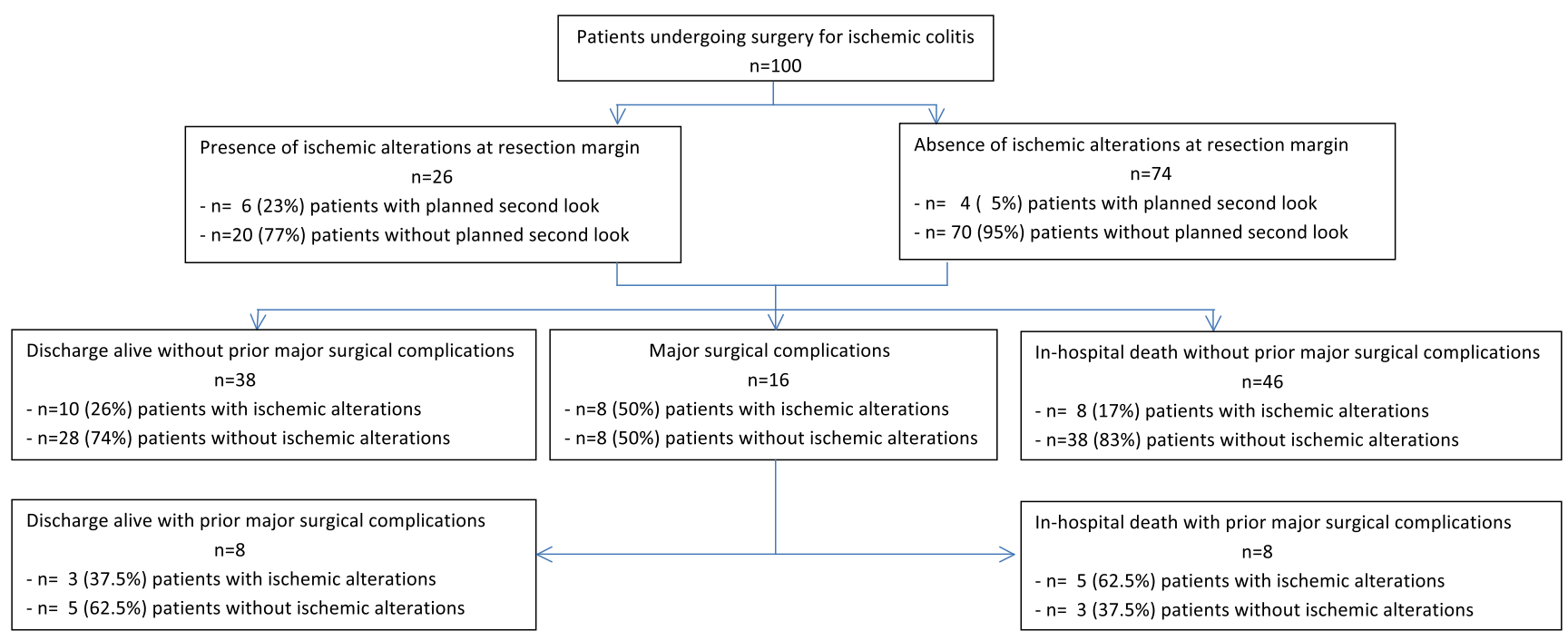

Fig. 1 Flow chart of patients and their respective outcomes $(n=100)$

replications carried out, and that its relationship with outcome is linear (variable transformation occurred only in $4 \%$ of all the occasions where the preoperative $\mathrm{pH}$ level was selected) (Supplementary Table 2a). Overall, the bootstrap analysis supported the choice of variables in our primary multivariable analysis: preoperative $\mathrm{pH}$ level, side of colon ischemia, and prior cardiac or aortic surgery, which were all selected in the primary analysis, were selected much more often in the bootstrap analysis than patients' age and gender, which were not selected in the primary analysis.

For patients who died in the hospital and those who were discharged alive from the hospital, reoperation for an elective second look was carried out in $8(15 \%)$ and $2(4 \%)$, respectively, and surgical revision for a complication was necessary in $22(41 \%)$ and $14(30 \%)$, respectively. One patient with rectal stump or stoma complications died without having had a reoperation.

Risk factors for major postoperative surgical complications

Characteristics of patients undergoing surgery for IC grouped by the occurrence of major surgical postoperative complications are shown in Table 2. Relative to patients who did not experience major postoperative surgical complications, patients who experienced major postoperative surgical complications were more likely to have ischemic alterations at the resection margin of the histological specimen. Additional patients' characteristics are shown in Supplementary Table 1b, where patients with major postoperative surgical complications were more likely to have COPD or acute renal failure.
Table 2 Characteristics of patients undergoing surgery for ischemic colitis (IC)

\begin{tabular}{lll}
\hline By in-hospital mortality & & \\
\hline Characteristic & Hospital death $(n=54)$ & Discharge alive $(n=46)$ \\
\hline $\begin{array}{l}\text { Median age (IQR), years } \\
\text { Female gender, } n(\%)\end{array}$ & $73(66,79)$ & $75(67,77)$ \\
Side of colon ischemia, $n(\%)$ & $20(37)$ & $20(43)$ \\
$\quad 13(24)$ & $27(59)$ \\
$\quad$ Left & $20(37)$ & $13(28)$ \\
$\quad$ Right & $21(39)$ & $6(13)$ \\
$\quad$ Pan-ischemia & $7.25(7.2,7.35)$ & $7.365(7.32,7.42)$ \\
$\begin{array}{l}\text { Median preoperative pH level (IQR) } \\
\text { Prior cardiac or aortic surgery, } n(\%)\end{array}$ & $20(37)$ & $13(28)$ \\
By major postoperative surgical complications & & No major postoperative surgical \\
Characteristic & Major postoperative surgical & complications $(n=84)$ \\
& complications $(n=16)$ & $75(67,80)$ \\
Median age (IQR), years & $71(65,75)$ & $18(21)$ \\
Histological specimen: ischemic alterations & $8(50)$ &
\end{tabular}


In the multivariable analysis, major postoperative surgical complications were more likely in patients with ischemic alterations at the resection margin of the histological specimen (OR 3.7; $95 \%$ CI 1.2, 11; $P=0.022$ ), whereas age was not found to be an independent important predictor for major postoperative surgical complications (Table 3).

The bootstrap analysis confirmed the results of the primary multivariable analysis, since ischemic alterations at the resection margin of the histological specimen showed a bootstrap inclusion frequency of $76 \%$ as compared with $31 \%$ for age (Supplementary Table 2b).

\section{Competing risks analysis}

The cumulative incidence functions for the two competing events hospital death and discharge alive are shown in Fig. 2, where the risk factors gender, side of ischemia, prior cardiac or aortic surgery, age, and $\mathrm{pH}$ level were considered in Fig. 2a, e.

The competing risk model explains how the different factors contribute to in-hospital mortality (Supplementary Table 3). Patients with a decreased preoperative $\mathrm{pH}$ level, with right-sided or pan-colonic ischemia, both relative to left-sided ischemia, and with prior cardiac or aortic surgery were more likely to die in the hospital and less likely to be discharged alive from the hospital. That is, these patients stayed longer in the hospital, and each day, they were exposed to the (significantly) increased risk of death. Ultimately, there were therefore markedly more such patients who died in the hospital. Since male patients stayed longer in the hospital and each day they were exposed to the (not significantly altered) risk of death, there were, relative to female patients, more male patients who died in the hospital. The corresponding cause-specific hazard ratios (with $95 \%$ confidence intervals) estimated in the multivariable analysis are shown in Supplementary Table 3.

Table 3 Odds ratios, $95 \%$ confidence intervals, and $P$ values of the multivariable fractional polynomials (MFP) model with variables and functional forms selected at the 0.2 level

Risk factors for in-hospital mortality

\begin{tabular}{llll}
\hline Risk factor & Odds ratio & $95 \% \mathrm{CI}$ & $P$ value \\
\hline $\begin{array}{llll}\text { Side of colon ischemia } \\
\quad\end{array}$ & 3.8 & $1.2,12$ & 0.022 \\
$\quad$ Right & 11 & $2.8,39$ & $<0.001$ \\
$\quad$ Pan-ischemia & 2.5 & $1.5,4.1$ & $<0.001$ \\
Preoperative pH level, decrease per 0.1 & 2.4 & $0.82,6.8$ & 0.109 \\
Prior cardiac or aortic surgery & 2.4 & & \\
Risk factors for major postoperative surgical complications & \\
$\begin{array}{l}\text { Histological specimen: ischemic } \\
\quad \text { alterations at the resection margin }\end{array}$ & 3.7 & $1.2,11$ & 0.022 \\
\hline
\end{tabular}

$C I$ confidence interval

\section{Discussion}

This study shows that colonic resection for IC is associated with high in-hospital mortality, especially in patients with a decreased preoperative $\mathrm{pH}$ level, with right-sided or pancolonic ischemia and after cardiac or aortic surgery, whereas age and gender were not found to be independent important predictors for hospital death. There is evidence that patients with ischemic alterations at the resection margin of the histological specimen are more likely to experience major postoperative surgical complications.

Relation to other studies investigating mortality after IC

Besides ours, only few studies focused solely on surgically treated patients $[14,15,22,23]$. In the literature, overall mortality rates of around $50 \%$ and up to $75 \%$ in total colonic ischemia have been reported [24, 25]. The overall in-hospital mortality of $54 \%$ in our series was similar, with a high amount of emergency operations ( $80 \%)$. Patients with pan-ischemia showed the highest in-hospital mortality (78 \% in our series).

We found hospital death to be more likely not only in patients with pan-colonic ischemia, but also in patients with right-sided as compared to left-sided ischemia. There are only few studies so far reporting a difference in mortality depending on the site of ischemia [26-29]. Sotiriadis and colleagues observed an overall mortality of $22.5 \%$ in right-sided versus $11.9 \%$ in left-sided colonic ischemia, including those patients who died before surgery; postoperative mortality rates were similar in both groups (37.5\% in right-sided versus $42.9 \%$ in left-sided colonic ischemia) [26]. The results of Brandt and colleagues showed a significantly increased risk for mortality in patients with rightsided ischemia compared to all other ischemic sites (left, transverse, distal colon; 20 versus $9 \%$ ), in a cohort of medically and surgically treated patients [27]. An investigation based on a prospective database provided evidence that total colectomy is a significant risk factor for mortality after surgery in univariate analysis only, but not in the multivariable analysis [15].

Several patient- and surgery-related characteristics other than referring to the site of ischemia have been previously found to be associated with in-hospital mortality in patients undergoing surgery for IC: preoperative lactate level, ASA classification, emergency surgery, acute renal failure, elevated intraoperative blood loss and allogeneic blood and fresh frozen plasma transfusions, occlusion of mesenteric artery, and low output heart failure or catecholamine administration [14, 15]. Serum lactate is generally known to be elevated in IC [14, $30,31]$. A preoperative increase of lactate $>2.5 \mathrm{mmol} / \mathrm{l}$ has previously been found to be associated with a significantly elevated mortality $[14,32]$. In our study, a median of $4.5 \mathrm{mmol} / \mathrm{l}$ (IQR $2.5,7.4$ ) was measured preoperatively in patients who died in the hospital versus a median of $2.1 \mathrm{mmol} / \mathrm{l}$ (IQR 1.1, 2.5) in patients leaving hospital alive. 
In bowel ischemia, decreased $\mathrm{pH}$ level has been shown to be a predictor for mortality [33, 34]. Accordingly, in the multivariable analysis, we identified a decreased preoperative $\mathrm{pH}$ level to be an important predictor for in-hospital mortality in patients treated surgically for IC.

There are several reports on IC after cardiac or aortic surgery procedures $[14,35-44]$. In the cited studies, mortality rates of $16-100 \%$ were found. Similarly, in our study, patients after cardiac or aortic surgery were more likely to die in the hospital. However, awareness regarding these diseases has grown and improvements in intensive care units have been made in recent years.

a

Male $(n=60)$

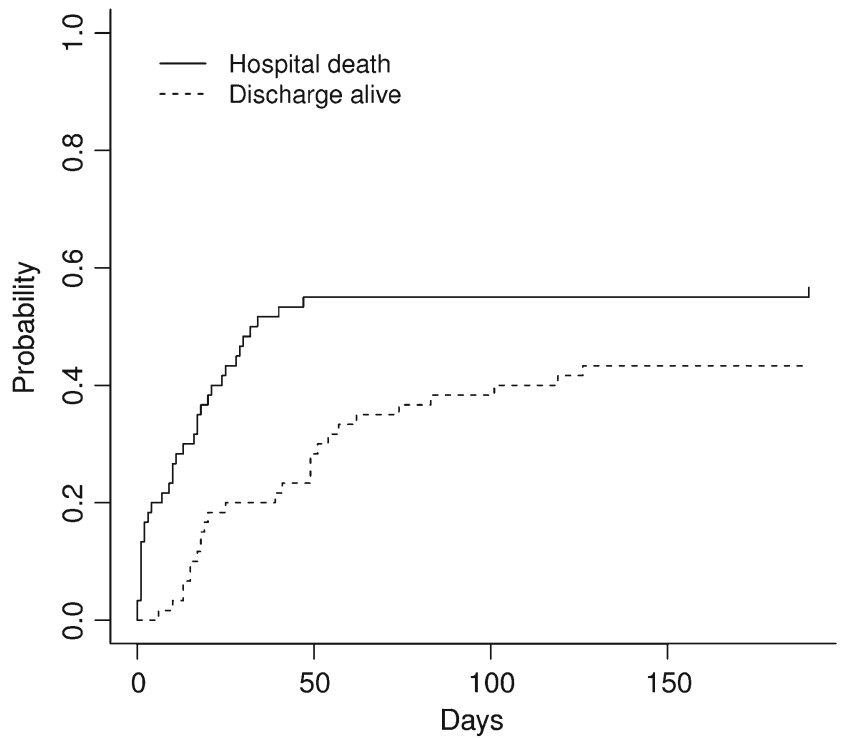

Relation to other studies investigating severe surgical complications after IC

Some authors suggest performing primary anastomosis after resection of ischemic colon only as an exemption due to the high risk of leakage [45]. This critical attitude towards primary anastomosis is supported by our data, with a leakage rate of $21 \%$ subsequently leading to a Hartmann procedure. But even after a Hartmann procedure as initial intervention, adverse outcomes are not uncommon. Thirteen percent of the patients in our series developed a necrosis of the stoma or an insufficiency of the rectal stump. In the

Female $(n=40)$

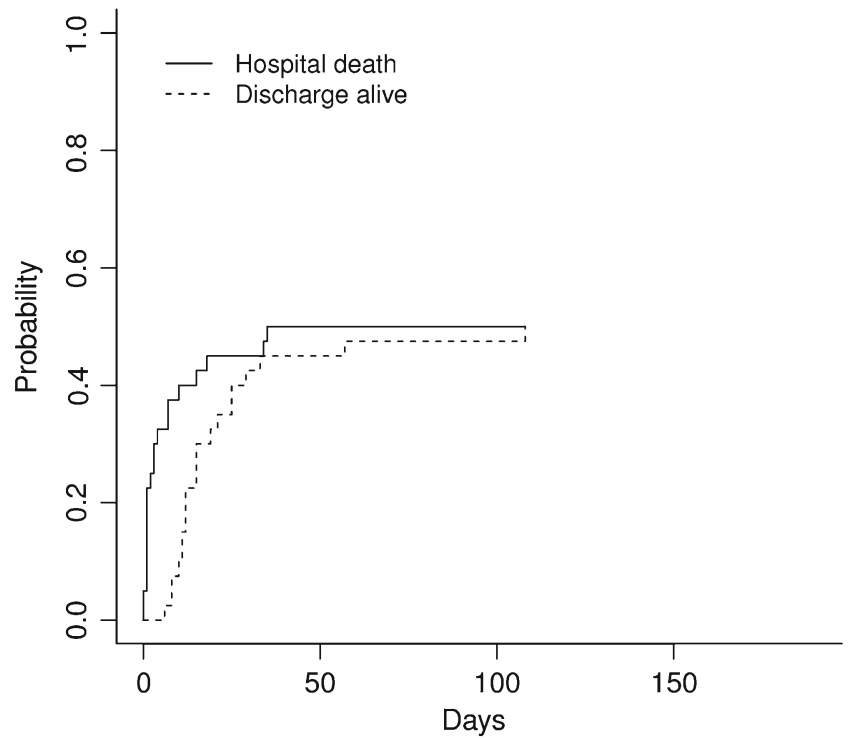

b
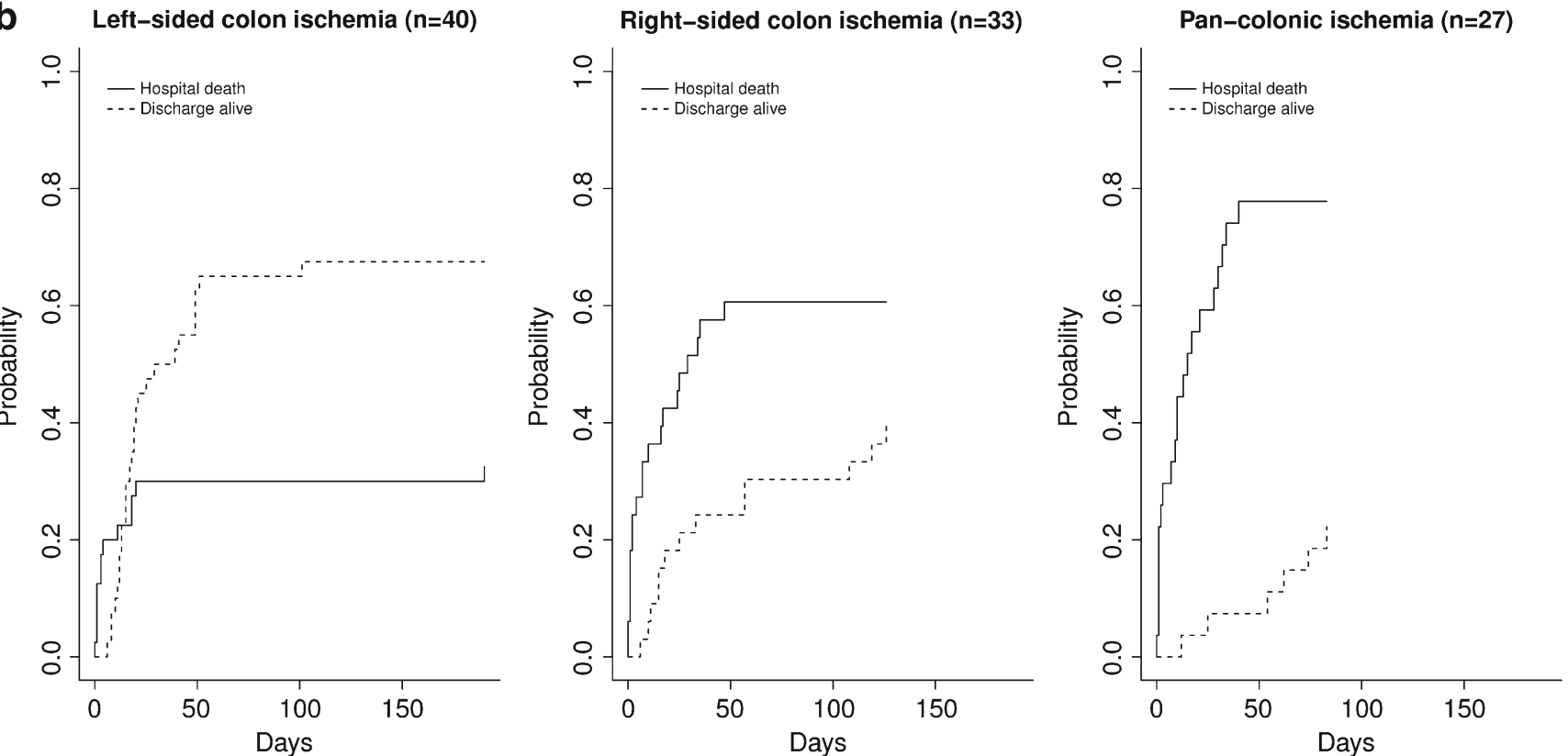

Fig. 2 Cumulative incidence functions for in-hospital mortality and discharge alive $\mathbf{a}$ by gender, $\mathbf{b}$ by side of colon ischemia, $\mathbf{c}$ by prior cardiac or aortic surgery, $\mathbf{d}$ by age (median split, $<74$ years versus $=74$ years), and $\mathbf{e}$ by $\mathrm{pH}$ level ( $\mathrm{pH}<7.38$ (acidosis) versus $\mathrm{pH}=7.38$ (no acidosis)) 

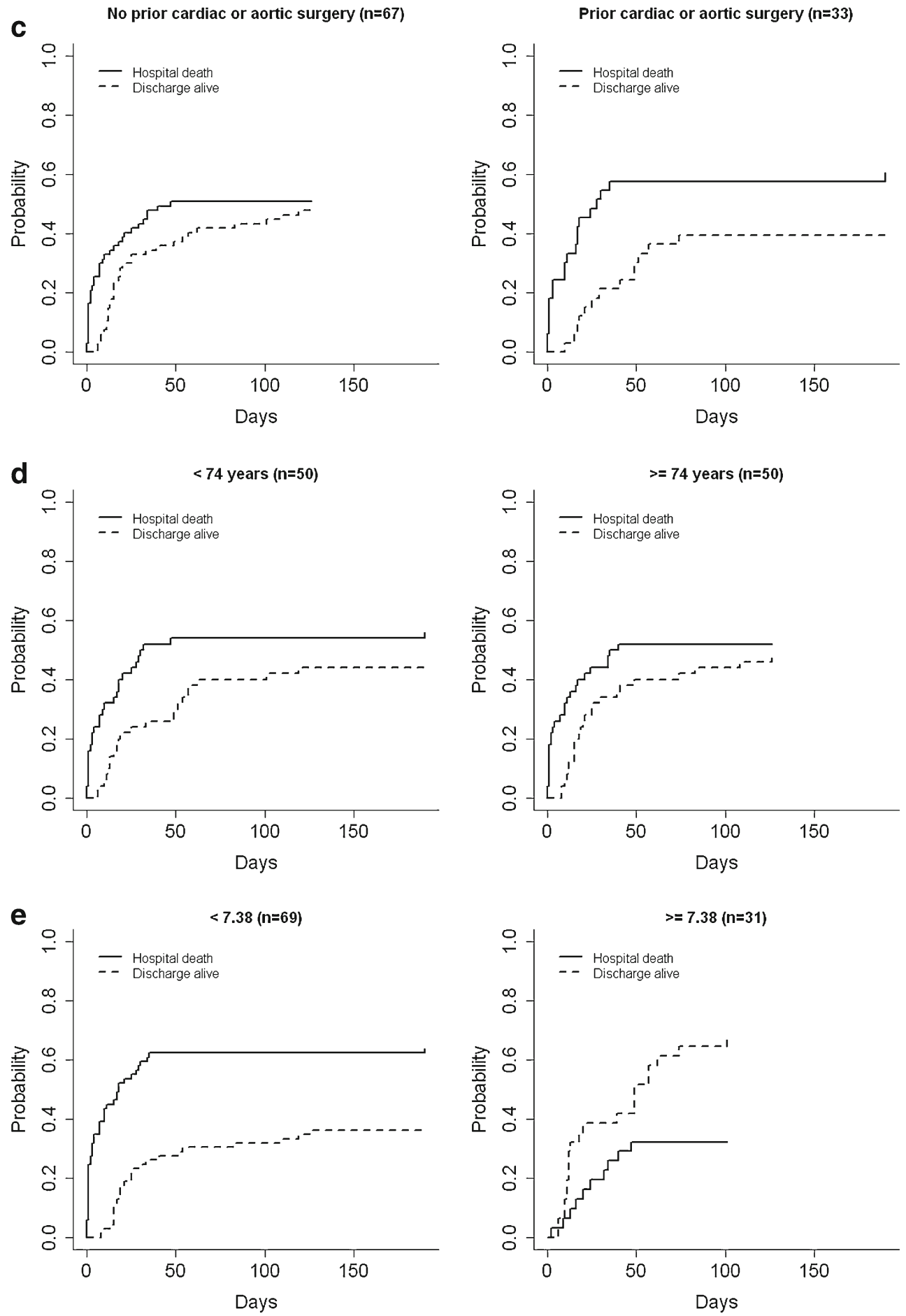

Fig. 2 (continued)

literature, stoma necrosis in IC is described with frequencies up to $50 \%$ [46].

However, to our knowledge, there is no study so far having analyzed the histological specimen under the aspect of ischemic alterations at the resection margins and having evaluated its association with major surgical complications. Our results suggest that margins' affection seems to be underestimated upon surgery and that there is a significantly elevated leakage and stoma complication rate for those patients.

Possible mechanisms explaining the findings

IC is generally viewed as a nonocclusive form of intestinal ischemia, e.g., during low-flow state in the course of cardiac 
surgery. The right colon may be vulnerable especially in this case, as the marginal artery of Drummond (system of vessel arcades connecting the major mesenteric arteries) is poorly developed here in $50 \%$ of the population [47]. In some cases, a specific anatomic abnormality may be identified, e.g., ligation or compromise in blood flow of the inferior mesenteric artery during abdominal aortic surgery. In case of poor blood supply through the anastomosis of Riolan, ischemia of the left colon will occur. In general, the pathology of ischemia in patients undergoing surgery seems to be different to that in patients suffering from chronic ischemic colitis.

As outlined above, the association of decreased preoperative $\mathrm{pH}$ level and mortality is an expected finding, whereas age not being an important predictor was rather surprising. A possible explanation is the median age of 74 in our entire cohort with quite a small interquartile range, so representing a rather homogenous age group. However, the data need to be interpreted with care, since the cause-specific hazard ratios were indicated per 10 years of increase, while the range of age was quite small as outlined above. Furthermore, the extent of atherosclerosis in the vessel arcades of the colon, which presumably increases with age, rather leads to a chronic state of ischemic colitis with recurrence of pain and difficulties with bowel movement than to fulminant IC. This might be a reason why we did not find age to be an independent predictor of death compared to studies focusing on all patients with IC [5].

The fact that patients with ischemic alterations at the resection margin of the histological specimen were more likely to have major postoperative surgical complications is an expected finding, suggesting that the presence of critical perfusion seems to be underestimated upon surgery. This is supported by a study describing the surgeons' predictive accuracy for anastomotic leakage in 191 patients undergoing colorectal resection with anastomosis to be low [48].

\section{Limitations of the study}

In accordance with studies in the field, the major limitations of our analysis are the retrospective study design and the limited number of purely surgically treated patients with IC despite the 7-year interval. In our statistical models, we respected this limitation by choosing the number of candidate predictors to be considered (five concerning mortality and two concerning major surgical complications) based on the number of hospital deaths and major postoperative surgical complications to avoid overfitting.

Another limitation is the fact that we assessed ischemic alterations at the resection margin as a risk factor for major surgical complications, but not for in-hospital mortality. We plan a larger follow-up study to further assess the effect of ischemic alterations on major surgical complications as well as major surgical complications and their effect on the further course of the disease (either hospital death or discharge alive).
Of note, patients who died in the hospital with no prior major surgical complications might have developed such complications, had they not died from other causes before.

\section{Strengths of the study}

All candidate predictors for in-hospital mortality considered in our analysis were defined in advance. The stability of our model was assessed with a bootstrap analysis, confirming the results. In an additional time-to-event analysis, we considered the two competing events of hospital death and discharge alive from the hospital.

To the best of our knowledge, ischemic alterations at the resection margins have not been previously investigated as risk factor for major surgical complications.

\section{Implications for daily practice}

From our point of view, there are several points to consider in the surgical management of ischemic colitis. (a) Concerning the type of intervention, discontinuity resection may be the safer choice of procedure in doubt of sufficient perfusion and in critically ill patients to allow early transfer to the ICU; however, our observational study - retrospective in naturemay not answer this question given the potential confounding by indication. Primary anastomosis can be performed, when blood supply of the residual colon and the formed anastomosis seems to be sufficient in the eyes and hands of an experienced surgeon and in case the patient is hemodynamically stable. (b) As for the choice of extension of resection, based on the high rate of major surgical complications and the finding that resection margins' affection seems to be underestimated upon surgery, resections should be carried out wide enough within healthy tissue. Devices to assess microperfusion may become an important adjunct to clinical judgment of intestinal perfusion. In addition to the assessment of intestinal perfusion based on color of the colonic wall and pulsation of vessel arcades patients' characteristics, pre- and other intraoperative findings need to be considered to estimate the risk of future surgical complications and to determine the extent of resection. (c) Importantly, the indication for a second look laparotomy should be put in place liberally. (d) Last, patients at risk to develop colonic ischemia, such as patients after cardiac and aortic surgery, should be closely monitored after these interventions and explorative abdominal surgery should be carried out liberally.

Implications for further research

Large prospective multicenter cohort studies are needed to assess more candidate predictors of mortality and surgical complications in patients undergoing surgery for IC. Our findings suggest a number of potential predictors, for instance 
ASA, small gut ischemia, or acute renal failure. A larger cohort of patients will allow including more of these more obvious predictors to be able to adjust for them while exploring predictors which have not yet been studied extensively. One of them is ischemic alterations at the resection margin, one of the most important findings of this study. This needs to be further studied using appropriate statistical methods that consider (1) death with no prior surgical complications as a competing event for the occurrence of surgical complications and (2) the time-dependent nature of the occurrence of surgical complications as a predictor of further course of the disease.

In randomized controlled trials, different treatment strategies should be evaluated such as screening colonoscopy, primary anastomosis with diverting stoma versus Hartmann procedures, the impact of scheduled second looks, and the use of devices determining microcirculation upon surgery. Methods like tissue photospectrometry could be implemented in determining intestinal venous oxygen saturation. As long as the oxygen saturation exceeds $10 \%$, the tissue is not considered hypoxic [49]. This method is used, e.g., to determine the level of extremity amputation in peripheral arterial vascular disease patients [50]. In plastic surgery, it has been used to control microvascular implants intra- and postoperatively [51]. In colorectal surgery, there are promising results in using spectroscopic devices in order to assess microperfusion in the anastomotic region and to predict anastomotic leakage [52-54]. The sample sizes of these studies are however fairly small, the interventions limited to elective surgery, there are no randomized controlled studies, and a clear cutoff level of oxygen saturation that would have as consequence an immediate follow-up resection or second look operation could not be determined so far. One could even think of an instantaneous section study to detect ischemic alterations at the resection margin, although this might not be practicable for the lack of infrastructure in most institutions.

The ultimate goal of the proposed surgical practice and further research is to reduce the high morbidity and mortality of patients with surgically treated IC.

\section{References}

1. Boley SJ, Schwartz S, Lash J et al (1963) Reversible vascular occlusion of the colon. Surg Gynecol Obstet 116:53-60

2. Greenwald DA, Brandt LJ (1998) Colonic ischemia. J Clin Gastroenterol 27(2):122-128

3. Thoedoropoulou A, Koutroubakis IE (2008) Ischemic colitis: clinical practice in diagnosis and treatment. World J Gastroenterol 14(48): 7302-7308

4. Chung JW, Cheon JH, Park JJ et al (2010) Development and validation of a novel prognostic scoring model for ischemic colitis. Dis Colon Rectum 53(9):1287-1294
5. Higgins PD, Davis KJ, Laine L (2004) Systematic review: the epidemiology of ischaemic colitis. Aliment Pharmacol Ther 19(7):729738

6. Brandt LJ, Boley SJ (1992) Colonic ischemia. Surg Clin North Am 72:203-229

7. Wilke R, Hutmacher J, Nowak T et al (2006) Chronic mesenteric ischemia with consecutive ischemic colitis. Suggestions for diagnosis and therapy. Chirurg 77(12):1152-1157

8. Feldman M, Friedman LS, Sleisenger MH (2002) Sleisenger and Fordtran's gastrointestinal and liver disease: pathophysiology, diagnosis, management, 7th edn. Saunders, Philadelphia

9. Brandt LJ (2006) Intestinal ischemia. In: Feldman M, Friedman L, Brandt LJ (eds) Sleisenger \& Fordtran's gastrointestinal and liver disease: pathophysiology, diagnosis, management. Elsevier, Philadelphia, pp 2563-2586

10. Baixauli J, Kiran RP, Delaney CP (2003) Investigation and management of ischemic colitis. Cleve Clin J Med 70:920-921, 925-926, 928-930 passim

11. Ritz JP, Germer CT, Buhr HJ (2005) Prognostic factors for mesenteric infarction: multivariate analysis of 187 patients with regard to patient age. Ann Vasc Surg 19:328-334

12. Brandt LJ, Boley SJ (2000) AGA technical review on intestinal ischemia. American Gastrointestinal Association. Gastroenterology 118:954-968

13. O'Neill S, Yalamarthi S (2012) Systematic review of the management of ischaemic colitis. Colorectal Dis 14(11):e751-e763

14. Reissfelder C, Sweiti H, Antolovic D et al (2011) Ischemic colitis: who will survive? Surgery 149(4):585-592

15. Antolovic D, Koch M, Hinz U et al (2008) Ischemic colitis: analysis of risk factors for postoperative mortality. Langenbecks Arch Surg 393(4):507-512

16. Peduzzi P, Concato J, Kemper E et al (1996) A simulation study of the number of events per variable in logistic regression analysis. J Clin Epidemiol 49:1373-1379

17. Sauerbrei W, Royston P (2002) Building multivariable prognostic and diagnostic models: transformation of the predictors by using fractional polynomials. J R Stat Soc Ser A (Stat Soc) 162:71-94, Corrigendum 165: 339-400

18. Sauerbrei W, Royston P, Binder H (2007) Selection of important variables and determination of functional form for continuous predictors in multivariable model building. Statist Med 26:5512-5528

19. Royston P, Sauerbrei W (2003) Stability of multivariable fractional polynomial models with selection of variables and transformations: a bootstrap investigation. Stat Med 22:639-659

20. Wolkewitz M, Vonberg RP, Grundmann H et al (2008) Risk factors for the development of nosocomial pneumonia and mortality on intensive care units: application of competing risks models. Critical Care 12:R44

21. Cox DR (1972) Regression models and life-tables. J R Stat Soc Ser B (Methodol) 34:187-220

22. Mentha G, Robert J, Rohner A (1990) Results of surgical treatment of ischemic colitis. Hel Chir Acta 57:777-782

23. Guivarc'h M, Roullet-Audy JC, Mosnier H et al (1997) Ischemic colitis. A surgical series of 88 cases. J Chir (Paris) 134:103-108

24. Longo WE, Ballantyne GH, Gusberg RJ (1992) IC: patterns and prognosis. Dis Colon Rectum 35:726-730

25. Longo WE, Ward D, Vernava AM 3rd et al (1997) Outcome of patients with total colonic ischemia. Dis Colon Rectum 40(12): 1448-1454

26. Sotiriadis J, Brandt LJ, Behin DS et al (2007) IC has a worse prognosis when isolated to the right side of the colon. Am J Gastroenterol 102(10):2247-2252

27. Brandt LJ, Feuerstadt P, Blaszka MC (2010) Anatomic patterns, patient characteristics, and clinical outcomes in IC: a study of 313 
cases supported by histology. Am J Gastroenterol 105(10): 2245-2252

28. Medina C, Vilaseca J, Videla S et al (2004) Outcome of patients with ischemic colitis: review of fifty-three cases. Dis Colon Rectum 47(2): 180-184

29. Flobert C, Cellier C, Berger A et al (2000) Right colonic involvement is associated with severe forms of ischemic colitis and occurs frequently in patients with chronic renal failure requiring hemodialysis. Am J Gastroenterol 95(1):195-198

30. Kaleya RN, Boley SJ (1995) Acute mesenteric ischemia. Crit Care Clin 11:479-512

31. Murray MJ, Gonze MD, Nowak LR et al (1994) Serum D(-)-lactate levels as an aid to diagnosing acute intestinal ischemia. Am J Surg 167:575-578

32. Gunnerson KJ, Saul M, He S, Kellum JA (2006) Lactate versus nonlactate metabolic acidosis: a retrospective outcome evaluation of critically ill patients. Crit Care 10(1):R22

33. Acosta-Merida MA, Marchena-Gomez J, Hemmersbach-Miller M et al (2006) Identification of risk factors for perioperative mortality in acute mesenteric ischemia. World J Surg 30(8):1579-1585

34. Scharff JR, Longo WE, Vartanian SM et al (2003) Ischemic colitis: spectrum of disease and outcome. Surgery 134(4):624-629, discussion 629-30

35. Welling RE, Roedersheimer LR, Arbaugh JJ et al (1985) Ischemic colitis following repair of ruptured abdominal aortic surgery. Arch Surg 120(12): 1368-1370

36. Maupin GE, Rimar SD, Villalba M (1989) Ischemic colitis following abdominal aortic reconstruction. Am Surg 55(6):378-380

37. Valentine RJ, Hagino RT, Jackson MR et al (1998) Gastrointestinal complications after aortic surgery. J Vasc Surg 28(3):404-411, discussion 411-2

38. Björck M, Troëng T, Bergqvist D (1997) Risk factors for intestinal ischeaemia after aortoiliac surgery: a combined cohort and case-control study of 2824 operations. Eur J Vasc Endovasc Surg 13(6):531-539

39. Darras S, Paineau J, Patra P et al (2011) Prognostic factors of ischemic colitis after infrarenal aortic surgery. Ann Vasc Surg 25(5):612-619

40. Gauss A, Drück A, Hemmer W et al (1994) Abdominal complications following heart surgery. Intensivmed Notfallmed Schmerzther 29(1):23-29

41. Gennaro M, Ascer E, Matano R et al (1993) Acute mesenteric ischemia after cardiopulmonary bypass. Am J Surg 166(2):231-236
42. Kurt M, Litmathe J, Roehrborn A et al (2006) Abdominal complications following open-heart surgery: a report of 12 cases and review of the literature. Acta Cardiol 61(3):301-306

43. Schütz A, Eichinger W, Breuer M et al (1998) Acute mesenteric ischemia after open heart surgery. Angiology 49(4):267273

44. Abboud B, Daher R, Boujaoude J (2008) Acute mesenteric ischemia after cardio-pulmonary bypass surgery. World J Gastroenterol 14(35): $5361-5370$

45. Izbicki JR, Schneider CG, Kastl S (2003) Partielle Ischämien. Okklusive und nichtokklusive Darmischämie, ischämische Kolitis, systemischer Lupus erythematodes. Chirurg 74:413-418

46. Leenen LP, Kuypers JH (1989) Some factors influencing the outcome of stoma surgery. Dis Colon Rectum 32(6):500-504

47. Baixauli J, Kiran RP, Delaney CP (2003) Investigation and management of ischemic colitis. Cleve Clin J Med 70(11):920 934

48. Karliczek A, Harlaar NJ, Zeebregts CJ et al (2009) Surgeons lack predictive accuracy for anastomotic leakage in gastrointestinal surgery. Int J Colorectal Dis 24(5):569-576

49. Harrison DK, Newton DJ, McCollumm PT et al (1996) Lightguide spectrophotometry for the assessment of skin healing viability in critical limb ischaemia. Adv Exp Med Biol 388:45-51

50. Harrison DK, McCollum PT, Newton DJ et al (1995) Amputation level assessment using lightguide spectro-photometry. Prosthet Orthot Int 19:139-147

51. Hölzle F, Loffelbein DJ, Nolte D et al (2006) Free flap monitoring using simultaneous non-invasive laser Doppler flowmetry and tissue spectrophotometry. J Craniomaxillofac Surg 34:25-33

52. Karliczek A, Benaron DA, Baas PC et al (2008) Intraoperative assessment of microperfusion with visible light spectroscopy in esophageal and colorectal anastomoses. Eur Surg Res 41(3):303-311

53. Karliczek A, Benaron DA, Baas PC et al (2010) Intraoperative assessment of microperfusion with visible light spectroscopy for prediction of anastomotic leakage in colorectal anastomoses. Colorectal Dis 12(10):1018-1025

54. Hirano Y, Omura K, Tatsuzawa Y, Shimizu J et al (2006) Tissue oxygen saturation during colorectal surgery measured by nearinfrared spectroscopy: pilot study to predict anastomotic complications. World J Surg 30(3):457-461 